\title{
Influencia de la gamificación en diferentes cursos y tipos de alumno
}

Rafael R. Sola-Guirado ${ }^{\text {; }}$ Sergio Castro-Garcia ${ }^{\text {; }}$ Emilio Gonzalez-Sanchez ${ }^{a}$

${ }^{a}$ E.T.S.I.A.M. Universidad de Córdoba. Ed. Leonardo Da Vinci, Ctra. N IV, km. 396. 14014. Córdoba.

\begin{abstract}
Resumen
La gamificación web abre nuevas oportunidades en la enseñanza, especialmente en materias relacionadas con agricultura donde existe una desmotivación general en el aprendizaje de contenidos teóricos. En este trabajo se ha diseñado un test con Kahoot! como evaluación intermedia, sobre los contenidos de un mismo módulo de enseñanza, impartido en diferentes cursos (extensión, grado y postgrado). Se ha evaluado su impacto sobre el proceso de aprendizaje de diferentes tipos de alumnos (edad, estudios previos, experiencia profesional...) y su valoración según un cuestionario. Los resultados muestran que la herramienta es aún desconocida y que aumentó su motivación, especialmente para aquellos con estudios no universitarios. El 56\% percibe la herramienta de gran utilidad en el aula y el 48\% que debería usarse mucho en casa. Sin embargo, no la utilizarían para evaluación final. A mayor rango académico del curso impartido, peor fueron los aciertos en el test. Esto estuvo relacionado con su experiencia como agricultores, y señala la importancia de la práctica en el aprendizaje. Hubo una ligera relación negativa entre el número de aciertos con el tiempo de respuesta. En los bloques no tecnológicos, hubo diferencias entre los tipos de cursos.
\end{abstract}

Keywords: gamificación, agricultura, autoevaluación, tipo alumno

\begin{abstract}
The gaming tools opens up new opportunities in teaching, especially in subjects related to agriculture where there is a general demotivation for theoretical contents. A test has been designed by Kahoot! as an intermediate self-assessment, based on the same teaching module, for different courses (extension, university and master) It has been assessed the tool impact on the learning process of different types of students (age, previous studies, professional experience ...) and it has been evaluated according to a questionnaire. The results show that the tool is still unknown and that it increased its motivation, especially for those with non-university studies. $56 \%$ looked at the tool very useful in the classroom and $48 \%$ would use it at home. However, they would not use it to final evaluation purposes. The higher the academic rank of the course was, the lower were the successes in the test. This was related to their experience as farmers, and this point out the importance of the practice in the learning. There was a slight negative
\end{abstract}


relationship between the number of hits and the response time. In the nontechnological blocks, there were differences between the different courses.

Palabras clave: gaming, agriculture, self-assessment, student profile. 


\section{Introducción}

Los métodos de aprendizaje están en continua evolución, condicionados en gran parte por el modo de vida de la sociedad. Este hecho conlleva a la necesidad de innovación pedagógica para la actualización y mejora de la calidad de la enseñanza. Las metodologías empleadas por los docentes deben ir alineadas con las necesidades de los alumnos y del mercado que los demanda (Rompelman, 2000). En este sentido y en este momento, las nuevas tecnologías de la información y la comunicación habilitan unas potentes herramientas de fácil uso para alumnado.

Las técnicas de gamificación tecnológicas parecen ser un buen aliado en el actual paradigma de la Educación Superior. Entre las posibilidades que ofrecen, puede tener gran utilidad en uno de los grandes problemas docentes: despertar el interés del alumno y dotarle de motivación (Lee y Hammer, 2011). Para ello, el uso de estas herramientas en el aula debe adecuarse para que el alumno pueda sentir logros a medida que progresa (Fitz-Walter,. Tjondronegoro y Peta, 2011). Con ello, se conseguirá mantener su interés y hacerlo formar parte activa del aprendizaje, no sólo como elemento de escucha del docente.

Además, para que este aprendizaje se desempeñe con éxito es importante que alumno sepa comprender su evolución, mediante evaluaciones intermedias en el desempeño de las competencias que se pretende que alcancen (Christoforou y Yigit, 2008). La realización de exámenes tradicionales intermedios en clase puede llegar a ser un elemento desmotivador para el alumno y que requiere una gran labor de corrección para el docente. Sin embargo, las nuevas herramientas de gamificación permiten que el alumno pueda autoevaluarse en tiempo real y a su vez, el profesor pueda detectar la incidencia de aquellas partes que han de reforzarse y poder ayudar a aquellos alumnos que tenga mayores necesidades.

La combinación de estas autoevaluaciones con las nuevas tecnológicas existentes de gamificación en el aula, puede se llevada a cabo con herramientas como "Socrative", "Pinnion" o "Polleveryway". En este trabajo, se ha utilizado "KAHOOT!" por su uso sencillo y gratuita en la creación y aplicación de cuestionarios. El alumno responde en tiempo real y recibe instantáneamente los resultados junto con una puntuación, de modo similar a una competición, para generar una adecuada retroalimentación con el alumno o el docente.

Existen numerosos estudios de utilización de este tipo de herramientas en diversos ámbitos tan variados como la informática (Jaume-i-Capó et al., 2016), actividades físicas (PérezLópez et al. (2017), odontología (Pintor et al., 2014), idiomas (Zarzycka, 2014), etc. Sin embargo, su aplicación en materias relacionadas con la agricultura es muy escasa. En este campo, el modo de enseñanza difiere mucho por la falta de una tipología de alumnado y su diversidad en cuanto a edad, formación, experiencia y nacionalidad. De entre los cursos más extendidos en agricultura, se encuentran los de extensión agraria, de grado o de postgrado. Debido a su orientación final aplicada y práctica, el alumando tiene una desmotivación en la impartición de los contenidos teóricos. Esto deriva a que tengan un rendimiento bajo en los exámenes finales debido a la falta de estudio. 


\section{Objetivos}

Por todo ello, parece interesante introducir la gamificación informática en el aula en estos cursos de formación agraria y evaluar su incidencia en el alumnado. Así pues, proponemos la aplicación de un test gamificado, como autoevaluación intermedia, en una serie de cursos, tanto desde una perspectiva universitaria como de extensión agraria, con contenidos comunes de mecanización agrícola. El objetivo es evaluar el impacto de la herramamienta y el test sobre las diferentes tipologías de alumnado en los diferentes tipos de cursos.

\section{Desarrollo de la innovación}

Se ha impartido un módulo de enseñanza en español en diferentes modalidades, todos relacionados con la mecanización agraria del olivar: 1) Curso para técnicos en Instituto de Formación Agraria, 2) asignatura de Grado (Ingeniería Agroalimentaria y del Medio Rural) y 3) Asignatura de Máster (Olivicultura). En cada curso hubo 15 alumnos (45 en total) con diferentes características en formación previa (universitaria y no universitaria), edades, experiencia previa como agricultores (sí y no), grado de asistencia a clase (más de 75\%, entre $50-75 \%$ y menos de 50\%) y lengua materna (española y extranjera de otros países mediterráneos como Egipto, Italia, Israel, Irán y Turquía).

Se elaboró un test que contiene preguntas relacionadas con los contenidos que se han explicado en un módulo concreto de "mecanización agraria del olivar" (5 horas de clase). El test está basado en 5 bloques de 5 preguntas por bloque con los ámbitos costes olivar, mecanización del olivar, conceptos técnicos, maquinaria e innovación. Para cada pregunta dispusieron de 30 segundos y los enunciados de las mismas estaban equilibrados para resolverlos en un tiempo similar. El test se aplicó a través de la plataforma "KAHOOT!" que aplica los test como un juego colaborativo en tiempo real y de manera virtual mediante los Smartphones de los alumnos. El test permitía 4 respuestas posibles, de las cuales una de las mismas era claramente errónea para un experto en la materia. Al final de cada test la plataforma emitió un informe que permitió evaluar el rendimiento individual y grupal.

Por otro lado, para estudiar la influencia de esta herramienta en la docencia, los estudiantes completaron un cuestionario sobre el uso de la herramienta. Se ha estudiado el conocimiento previo de la herramienta, el grado de motivación que ha supuesto en ellos, el uso de la herramienta en la enseñanza y aprendizaje, la frecuencia que se estima adecuada para usarla, sus posibilidades en otro ámbito, su utilidad para el estudio y su comparación como herramienta de evaluación respecto a un test ordinario.

\section{Resultados obtenidos y discusión}

\section{Valoración de la herramienta por el alumno}

La edad de los alumnos estuvo entre 20 y 58 años con una mediana 24 años y una media de 27 años. 15 alumnos no tenían el español como lengua materna. 15 de los alumnos no tenían ninguna experiencia previa como agricultores. El 77\% de los mismos acudió a más de $4 \mathrm{~h}$ de las $5 \mathrm{~h}$ impartidas, el $20 \%$ a más de la mitad de las horas impartidas y el 3\% restante a menos de la mitad. El 20\% de los alumnos no tenía ningún tipo de estudios universitarios.

(c) ) EY-NC-ND 2018, Universitat Politècnica de València

Congreso IN-RED (2018) 
Los resultados del cuestionario reflejan que esta herramienta era muy desconocida en el ámbito de enseñanza agrario, no habiéndola utilizado nada en más del 75\% de los alumnos encuestados. A la mayoría de los estudiantes les pareció muy o bastante adecuada para despertar su atención, siendo aquellos a los que motivó mucho principalmente aquellos sin estudios universitarios (77,8 \%). En este ámbito de motivación, ninguno de los encuestados respondió de manera negativa coincidiendo con otros autores (Lee y Hammer, 2011, Teixes, 2015).

El 57\% de los alumnos considera muy útil el uso de la herramienta en el aula en este tipo de contenidos, siendo mejor la valoración en los cursos de extensión agraria $(3,8 / 4)$ que en los de ámbito universitario $(3,4 / 4)$. Sin embargo, el $64 \%$ consideran que su uso en el aula podría aumentar para utilizarla con bastante frecuencia, pero no mucho, especialmente en el grupo de cursos universitarios, donde este porcentaje sube al 75\%. Además, el 58\% de los alumnos de cursos no universitarios creen la gamificación les ha ayudado mucho para afianzar los conocimientos aprendidos frente al 34\% los cursos universitarios. Ello, indica que podría ser utilizada a la finalización de alguna unidad específica para afianzar conocimientos como señala Yapıcı y Karakoyun (2017). El hipotético uso de la herramienta fuera de clase como complemento al estudio, es considerado de bastante utilidad por el 55\% de los alumnos y de mucho potencial por el $45 \%$ restante. Esto sugiere el uso de la herramienta para el trabajo fuera del aula, permitiéndole estudiar o repasar de un modo más ludifico.

En el apartado del uso de la herramienta para la evaluación final frente a un examen test tradicional, se obtuvo una nota media más baja. El $44 \%$ de los alumnos de los cursos no universitarios piensan que este tipo de evaluación les ha perjudicado mucho o bastante en su rendimiento, con un porcentaje mayor en los cursos universitarios (56,7\%). En los grupos de grado, fue donde se obtuvo una peor nota de 2,2/4. Esto es explicado por otros autores por el efecto negativo de la competitividad y del tiempo en la realización del test (Glover, 2013.

En general, en ninguna de las respuestas ha habido una relación ni en el factor edad, ni en el factor lengua materna, con la valoración del cuestionario. Ello determina, que el uso de la gamificación no está influenciado por la edad, ni la generación de la que provenga, pudiendo extenderse su uso a cualquier ámbito en la enseñanza.

\section{Resultados del test en el ámbito agrícola entre los grupos de estudio.}

Los resultados de los test realizados a los alumnos muestran diferentes resultados para distintos grupos de alumnos (Figura 1). En primer lugar, el nivel de estudios no repercute positivamente en el número de aciertos, es más, alumnos con niveles universitarios obtuvieron una media de 2,6 aciertos menos que aquellos con estudios no universitarios. Sin embargo, hay que señalar que esto se explica puesto que el grupo sin estudios universitarios estaba compuesto por agricultores, con experiencia en el sector. La variable experiencia tiene un peso muy importante en los aciertos con diferencias significativas ( $\mathrm{p}<0,05$, t-student) entre los aciertos de los alumnos con experiencia previa $(\mathrm{n}=25)$ y $\sin$ ella. De esta manera, el número aciertos medios fue de 19,14 para los estudiantes sin 
estudios universitarios y agricultores ( $\mathrm{n}=10), 17,16$ para aquellos con niveles universitarios y agricultores $(n=19)$, y 15,64 para los universitarios sin experiencia previa $(n=15)$.

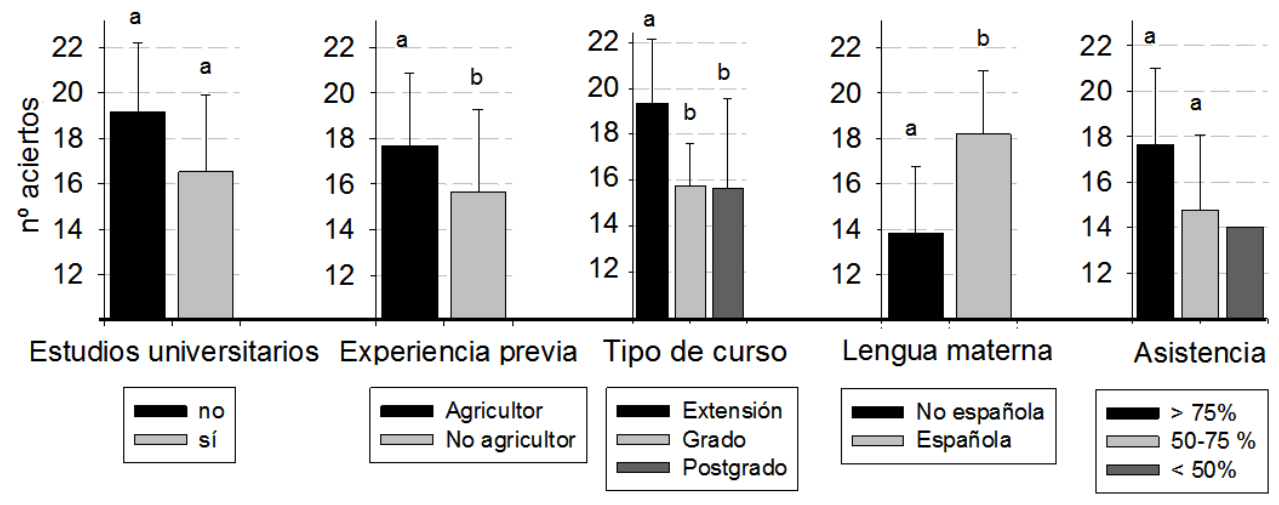

Fig. 1 Aciertos de los alumnos en el test según agrupación en diferentes categorías. Letras diferentes indican diferencias significativas entre barras para cada categoría ( $p<0.05$, test de Duncan para "Tipo de curso" y “Asistencia” y test $t$-student para el resto..

Se observa una tendencia decreciente del número de aciertos desde los cursos de mayor grado, con diferencias significativas entre los cursos de extensión y los universitarios ( $<<0.05$, Duncan test). De nuevo, la variable experiencia previa tiene cierta relación ya que, por lo general, el número de agricultores es mayor en cursos de menor rango académico: extensión $(n=16)$, grado $(n=6)$, postgrado $(n=6)$. Ha sido estudiado que la elección del alumno de una formación está altamente influenciada por su ámbito familiar (Esquivel, 1994; Martinez, 1993), siendo los alumnos provenientes de un ámbito rural los prefieren cursos de extensión y manifiestan mayor experiencia.

Entre cursos de grado y postgrado no se observan diferencias entre el número de aciertos, pero en postgrado la desviación es mayor. Ello puede ser en parte explicados por la variable lingüística, ya que en los cursos de postgrado había un mayor número de alumnos con lengua extranjera $(n=9)$ frente a los de grado $(n=2)$. Esta variable muestra diferencias significativas ( $<<0,001$, $t$-student) entre los aciertos de los grupos con habla española y extranjera. Esto refleja la barrera lingüística en la enseñanza (Sawir, E. (2005).

La relación entre el número de aciertos del alumno con su asistencia a clase, parece indicar una relación positiva (Figura 1), ya que los alumnos que asistieron que asistieron a más del $75 \%$ de las clases obtuvieron más de 2,9 aciertos con respecto a los que no lo hicieron. Ello refuerza la creencia de que la asistencia es un importante predictor del rendimiento del alumno (Credé et al., 2010).

El tiempo medio de los distintos estudiantes para responder fue de $12,89 \pm 2,85 \mathrm{~s}$. Si se analiza la influencia de los aciertos con el tiempo de respuesta, se observan ligeras diferencias entre los grupos estudiados. No existen diferencias entre las variables de agrupación, ya que sólo muestran diferencias entre grupos de menos de $1 \mathrm{~s}$, excepto en la variable lengua, donde existe una diferencia de casi 2,9 s. Existe una ligera tendencia que indica que el número de aciertos medio es inversamente al tiempo medio de respuesta

(cc) EY-NC-ND 2018, Universitat Politècnica de València

Congreso IN-RED (2018) 
(pendiente de $-11^{\circ}, \mathrm{R} 2=0,04$ ) y esto denota que el acierto puede estar relacionado con la seguridad del alumno al responder a la pregunta.

Finalmente, se observa que no existe una relación directa entre la edad del alumnado ni con el número de aciertos ni con el tiempo medio empleado en responder.

\section{Resultados de los alumnos por bloques}

El análisis de los aciertos entre los diferentes bloques del test indica diferencias entre los grupos estudiados (Figura 2). En el factor estudios universitarios no se observa ninguna diferencia significativa entre bloques. Esta tendencia es similar si los aciertos se agrupan por la experiencia previa, señalando en este caso, que en el bloque 4 de maquinaria, sí existe una diferencia significativa ( $\mathrm{p}<0,05$, $\mathrm{t}$-student) entre aquellos con y sin experiencia como agricultores. Las preguntas de este bloque, que versaron sobre identificar diferentes tipos de máquinas, reflejan cómo puede ser más eficaz iteractuar de manera práctica con una máquina, que sólo estudiarla apoyándose en fotografías. En la agrupación por tipo de curso, el número de aciertos en los bloques 1 y 4 (costes de olivar y maquinaria de olivar) fue significativamente diferentes ( $\mathrm{p}<0,05$, Duncan test) entre el curso de extensión y los de grado y postgrado, quizás debido a su carácter más generalista al igual que el planteamiento de los cursos de extensión. Sin embargo, en la agrupación por lengua materna, fueron los bloques 2 y 3 (mecanización y conceptos técnicos) los que supusieron diferencias significativas ( $\mathrm{p}<0,05$, t-student) entre los estudiantes de habla española, pudiendo explicarse a que el carácter más específico de estos bloques cuesta más su entendimiento en otra lengua.

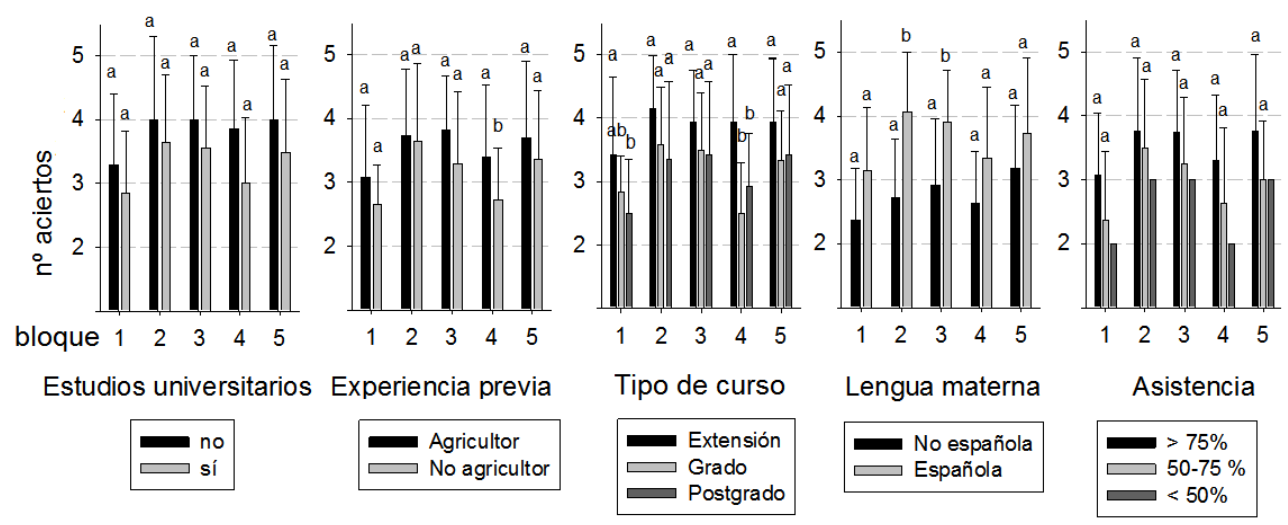

Fig. 2 Aciertos de los alumnos en el test según los distintos bloques (1 costes del olivar, 2 mecanización del olivar, 3 conceptos técnicos, 4 maquinaria e 5 innovación según agrupación en diferentes categorías. Letras diferentes indican diferencias significativas entre barras para cada categoría $(p<0.05$, test de Duncan para “Tipo de curso" y "Asistencia” y test $t$-student para el resto.

Si se analiza la incidencia del tiempo de respuesta según el bloque de preguntas (Figura 3) se observa un tiempo medio de $14,1 \pm 4,5 \mathrm{~s}$ para el bloque de costes de olivar, $15,2 \pm 3,71 \mathrm{~s}$ para el bloque de mecanización general, 13,4 $\pm 3,5 \mathrm{~s}$ para el bloque de conceptos técnicos, 11,3 \pm 3,6 s para el bloque de maquinaria y 10,46 \pm 3,3 s para el bloque de innovación. En general, el bloque 4 de maquinaria requirió menor tiempo que el resto, significativamente diferente ( $<<0,05$, Duncan test) entre el tipo de curso de extensión y el de postgrado. 
Igualmente, el bloque 5 de innovación requirió menos tiempo que el resto, coincidiendo además con el bloque con el mayor número de aciertos medios 3,7/5. Este último dato podría indicar que el alumno presta más atención a aquellos contenidos que hacen referencia al presente y futuro.

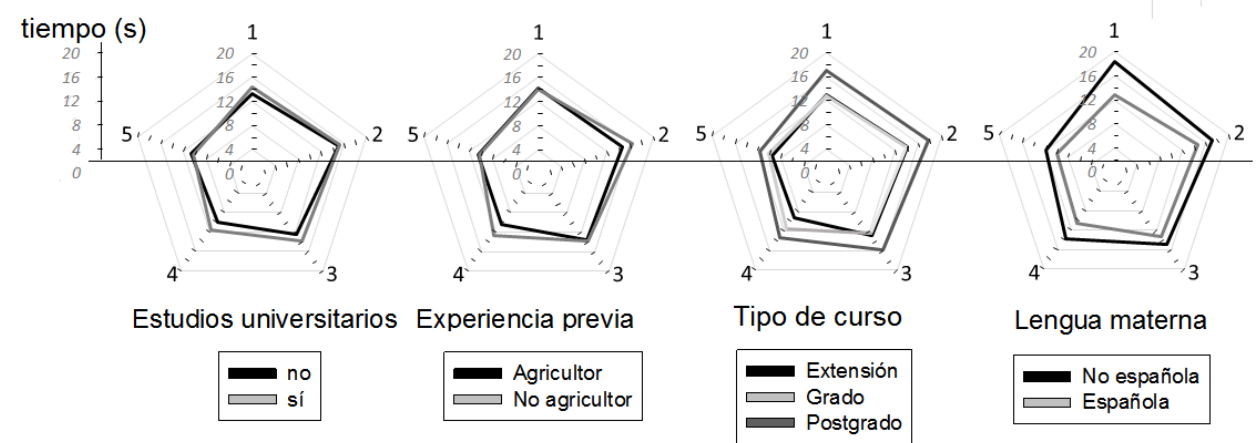

Fig. 3 Tiempo medio empleado de los alumnos en responder a las preguntas del test según los distintos bloques (1 costes del olivar, 2 mecanización del olivar, 3 conceptos técnicos, 4 maquinaria e 5 innovación según agrupación en diferentes categorías.

\section{Conclusiones}

La utilización de la gamificación en el aula de contenidos de agricultura ha tenido una fuerte aceptación a pesar de su desconocimiento previo. Los alumnos consideran que es un elemento motivador, que debería usarse frecuentemente como complemento a la enseñanza y que sirve de ayuda a la comprensión, especialmente los alumnos de cursos no universitarios. En general, no consideran adecuado su uso para la evaluación en exámenes finales.

Se ha puesto de manifiesto que la experiencia como agricultor es uno de los factores de mayor influencia para la resolución del test, y que en los cursos de extensión agraria es donde mayor número de alumnos existe con este perfil. La asistencia a clase también se muestra como indicador de buenos resultados. Existe un escollo con el idioma que repercute en peores resultados en los aciertos, donde en los bloques muy técnicos tienen un significativo menor número de aciertos con respecto a los alumnos de igual lengua a la de impartición de las clases.

Existe una ligera relación inversa entre el tiempo medio de contestación y el número de aciertos. No existen grandes diferencias entre las poblaciones de los diferentes grupos estudiados, pero parece que los bloques de maquinaria requieren de menor tiempo para la respuesta. Los bloques más generalistas de costes y maquinaria tienen mayor número de aciertos por los cursos más genéricos de extensión.

\section{Referencias}

CHRISTOFOROU, A. P., Y YIGIT, A. S. (2008). "Improving teaching and learning in engineering education through a continuous assessment process en European Journal of Engineering Education, 33(1), 105-116.

(cc) EY-NC-ND 2018, Universitat Politècnica de València

Congreso IN-RED (2018) 
CREDÉ, M., ROCH, S. G., KIESZCZYNKA, U. M. (2010). “Class attendance in college: A meta-analytic review of the relationship of class attendance with grades and student characteristics” en Review of Educational Research, 80(2), 272-295.

ESQUIVEL, L. (1994). " Toma de decisiones: Autoreporte de los factores que influyen en la elección de una carrera profesional." En Educación y ciencia, 39( 9), 37-53.

FITZ-WALTER Z., TJONDRONEGORO D. W. Y WYETH P. “Orientation Passport: using gamification to engage university students” en OzCHI 2011, Canberra, Australia. 2011.

GLOVER, I. (2013). "Play As You Learn: Gamification as a Technique for Motivating Learners" en World Conference on Educational Multimedia, Hypermedia and Telecommunications. Chesapeake: AACE.

JAUME-I-CAPÓ, A., LERA, I., VIVES, F. J., MOYÀ-ALCOVER, B., GUERRERO TOMÉ, C. (2016). "Experiencia piloto sobre el uso de la gamificación en estudios de grado de ingeniería en informática” en Simposio-Taller XXII JENUI, Universidad de Almería.

LEE J. Y HAMMER J. (2011). “Gamification in Education: What, How, Why Bother?” en Academic Exchange Quarterly, 15( 2).

MARTINEZ, T. (1993). "Familia y elección de carrera" en Perfiles educativos. Redalyc, 60,79-82.

PÉREZ-LÓPEZ, I. J., RIVERA GARCÍA, E., DELGADO-FERNÁNDEZ, M. (2017). "Mejora de hábitos de vida saludables en alumnos universitarios mediante una propuesta de gamificación” en Nutrición Hospitalaria, 34(4).

PINTOR HOLGUÍN, E., GARGANTILLA MADERA, P., HERREROS RUIZ VALDEPEÑAS, B., LÓPEZ DEL HIERRO CASADO, M. (2014). "Kahoot en docencia: una alternativa practica a los clickers” en XI Jornadas Internacionales de Innovación Universitaria.

ROMPELMAN, O. (2000). "Assessment of student learning: evolution of objectives in engineering education and the consequences for assessment" en European Journal of Engineering Education, 25(4), 339-350.

SAWIR, E. (2005). "Language difficulties of international students in Australia: The effects of prior learning experience” en International Education Journal, 6(5), 567-580.

TEIXES, F. (2015) Gamificación. Motivar jugando. Barcelona: Editorial UOC.

ZARZYCKA-PISKORZ, E. (2016). "Kahoot It or Not? Can Games Be Motivating in Learning Grammar?” in Teaching English with Technology, 16(3), 17-36. 